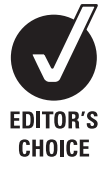

CHOICE

\title{
Effects of albendazole treatment on neurocysticercosis: a randomised controlled trial
}

\author{
A Carpio, ${ }^{1,2}$ E A Kelvin, ${ }^{2,3}$ E Bagiella, ${ }^{4}$ D Leslie, ${ }^{5,6}$ P Leon, ${ }^{7}$ H Andrews, ${ }^{3,4}$ W A Hauser, ${ }^{2,8}$ \\ the Ecuadorian Neurocysticercosis Group
}

\begin{abstract}
See Editorial Commentary, p 978

${ }^{1}$ School of Medicine, University of Cuenca, Cuenca, Ecuador;

${ }^{2}$ GH Sergievsky Center, College of Physicians and Surgeons, Columbia University, New York, USA; ${ }^{3}$ Data Coordinating Center at the New York State Psychiatric Institute and Columbia University, New York, USA: ${ }^{4}$ Department of

Biostatistics, Mailman School of Public Health, Columbia University, New York, USA;

${ }^{5}$ MRI Diagnostics of

Westchester, New York, USA:

${ }^{6}$ Good Samaritan Hospital,

Suffern, New York, USA;

${ }^{7}$ Instituto de Diagnóstico por Imágenes, Cuenca, Ecuador;

${ }^{8}$ Department of Epidemiology, Mailman School of Public Health, Columbia University, New York, USA
\end{abstract}

Correspondence to: W A Hauser, Gertrude H Sergievsky Center, Columbia University, 622 West 168th

Street, New York, NY 10032.

USA; wahausera@optonline.net

Received 18 January 2008 Revised 27 March 2008 Accepted 1 April 2008

Published Online First

21 May 2008

\section{ABSTRACT}

Aim: The aim of this trial was to evaluate the effects of albendazole $(A L B)$ on cyst disappearance, reduction of the number of cysts and seizure recurrence.

Methods: 178 patients with new onset symptoms due to active or transitional neurocysticercosis were randomly assigned to receive either $800 \mathrm{mg}$ of ALB daily or placebo for 8 days. All patients also received prednisone. Imaging studies were done at baseline and at months 1, 6 and 12 of follow-up.

Results: Active cysts were identified in 59 of 88 people randomised to ALB and 57 of the 90 in the placebo arm. By 1 month, 31\% were free of active cysts in the treatment group compared with $7 \%$ in the placebo group $(p=0.001)$. In addition, the ALB group had a greater reduction in the number of active cysts compared with the placebo group $(p=0.001)$. After 1 month following treatment there was no additional gain by treatment group in the disappearance or reduction in the number of active cysts. ALB treatment had little effect on cysts in the transitional or calcification stage. We found no difference between the ALB and placebo groups in symptoms during treatment or in seizure recurrence during the 12 months after treatment.

Conclusion: Albendazole plus symptomatic treatment leads to the disappearance of active cysts in $31 \%$ of patients compared with $7 \%$ of those with symptomatic treatment alone. This treatment effect occurs within the first 30 days after treatment.

Trial registration number: NCT00283699.

Neurocysticercosis (NC), the infection caused by the larval stage of the tapeworm Taenia solium, is the most frequently occurring parasitic disease affecting the human CNS. ${ }^{1}$ The disease is associated with clinical manifestations such as seizures, headache and focal neurological deficits. ${ }^{12} \mathrm{NC}$ is a serious public health problem for most of the developing world as well as for developed countries with high immigration rates from endemic countries in Latin America, Asia and Africa. ${ }^{2}$ NC has been designated as an emerging infection by the US Centers for Disease Control. ${ }^{34}$

A number of studies have been published related to treatment for NC with antihelminthic drugs (AHD), such as praziquantel and albendazole (ALB) ${ }^{5-10}$ Although treatment should cause earlier degeneration of cysticerci and could therefore decrease the risk of persistent neurological symptoms, $^{7}$ there are concerns that seizures and other neurological events can be triggered by the inflammatory reaction to treatment induced cysticercal degeneration. ${ }^{6}$ Frequently, spontaneous resolution of parenchymal cysticerci is observed on serial imaging studies. ${ }^{8}$ A systematic review of the literature stated that there is insufficient evidence to conclude that AHD treatment is associated with therapeutic benefit in $\mathrm{NC}^{9}$ and a recent metaanalysis of $\mathrm{NC}$ treatment concluded that there was evidence of only a modest effect of drug treatment in patients with NC. ${ }^{11}$ To date, there remains a debate over the value and safety of AHD therapy. ${ }^{9} 12$

This paper reports the results of a double blind, randomised, placebo controlled trial to evaluate the effects of ALB treatment on cyst disappearance, reduction in the number of cysts and seizure recurrence in patients with NC.

\section{METHODS}

\section{Participants}

Patients were recruited from six hospitals in Ecuador: three in Quito, two in Cuenca and one in Guayaquil. Approval for the study was granted by the Institutional Review Board of Columbia University, the Office for Human Research Protection (OHRP) of the National Institutes of Health in the USA, as well as the ethics committees at each of the participating hospitals.

Patients of any age or gender were eligible to participate if they had experienced new onset of symptoms associated with NC within 2 months prior to recruitment and had active and/or transitional NC cysts identified on axial CT or MRI of the brain. Non-inclusion criteria included having only calcifications, pregnancy, papilloedema, active tuberculosis, syphilis, ocular cysticercosis, active gastric ulcers or any progressive or life-threatening disorder. Patients who had received AHD during the year preceding presentation or who had received steroids within 30 days of presentation were also ineligible. Midway through the study, patients with ventricular shunt were excluded for safety concerns.

\section{Diagnostic criteria for neurocysticercosis}

A diagnosis of $\mathrm{NC}$ was made if a patient met any of the following criteria: ${ }^{13}$

1. One or more active parenchymal cysts: the CT scan shows circumscribed, rounded, hypodense areas without contrast enhancement. The MRI shows a CSF-like intensity signal on all sequences with no surrounding high signal. Both MRI and CT may show a high intensity or hyperdense $2-4 \mathrm{~mm}$ mural nodule depicting the scolex in the interior of the cyst.

2. One or more transition or degenerative parenchymal cysts: the CT scan shows annular contrast enhancement, surrounded by irregular 
perilesional oedema; there is a diffuse hypodense area with irregular borders on non-contrast CT, or a small, hyperdense, nodule surrounded by oedema. On MRI, the fluid content is of higher signal than CSF on T1 and T2 weighted images. The cyst capsule exhibits of low signal on the T2 images, is surrounded by oedema and enhances on gadolinium enhanced T1 weighted images.

3. Any of the above descriptions associated with an extraparenchymal location.

\section{Interventions}

For participants weighing more than $50 \mathrm{~kg}$, active treatment consisted of $400 \mathrm{mg}$ of ALB given orally every $12 \mathrm{~h}$ for 8 days. This dose has been used in other trials ${ }^{5}{ }^{14}$ and longer duration of treatment does not seem to have any advantage. ${ }^{14}$ For participants weighing less than $50 \mathrm{~kg}$ (including children), a dose of $15 \mathrm{mg} / \mathrm{kg} /$ day for 8 days was prescribed, as suggested by the drug manufacturers. ${ }^{15}$ The study pharmacist prepackaged bottles of 32 active drug ( $200 \mathrm{mg}$ ALB tablets) or 32 identical looking placebo tablets identified only by an assigned letter to maintain the blinding of study staff. The study nurse dispensed the drug in hospital and directly observed the patient taking the medication.

All patients received prednisone. Participants weighing $50 \mathrm{~kg}$ or more received $75 \mathrm{mg}$ of prednisone daily for 8 days, then $50 \mathrm{mg}$ /day for 1 week and finally $25 \mathrm{mg}$ /day for 1 week. Participants weighing less than $50 \mathrm{~kg}$ were prescribed $1.5 \mathrm{mg} /$ $\mathrm{kg} /$ day for 8 days, then $1 \mathrm{mg} / \mathrm{kg} /$ day for 1 week and finally
$0.5 \mathrm{mg} / \mathrm{kg} /$ day for 1 week. Patients with newly occurring seizures were prescribed phenytoin at standard doses. Carbamezapine was substituted if phenytoin was contraindicated or if seizure control was not achieved with phenytoin.

At enrolment, patients were interviewed to collect information on demographics and symptoms. In addition, a study related brain CT or MRI with and without contrast was taken within 2 weeks of enrolment.

During treatment, the study nurse monitored patients daily for adverse events, using a precoded symptom checklist. Information on symptoms was also collected at each followup visit in a similar manner. An independent safety monitoring committee was established to review the safety of all enrolled patients on an ongoing basis.

The research staff in Ecuador entered all data into a web based database using Scientific Web based Information Management Software (SBS Inc.), located on a server at Columbia University. The rate of data entry error was consistently found to be less than $2 \%$.

CT and MRI images were posted to a secure website from which they were accessible for reading by two neuroradiologists, one in Ecuador and one in the USA. Follow-up images were of the same type (CT or MRI) as the baseline image in order to make comparisons. Each scan was read independently, without knowledge of treatment arm or results of prior scans. Information collected from each scan included number of cysts of each phase (active, transitional and inactive calcification) by brain location. The inter-rater reliability of the readings was fair
Figure 1 Flow diagram of a multicentre trial comparing albendazole with placebo in patients with neurocysticercosis.

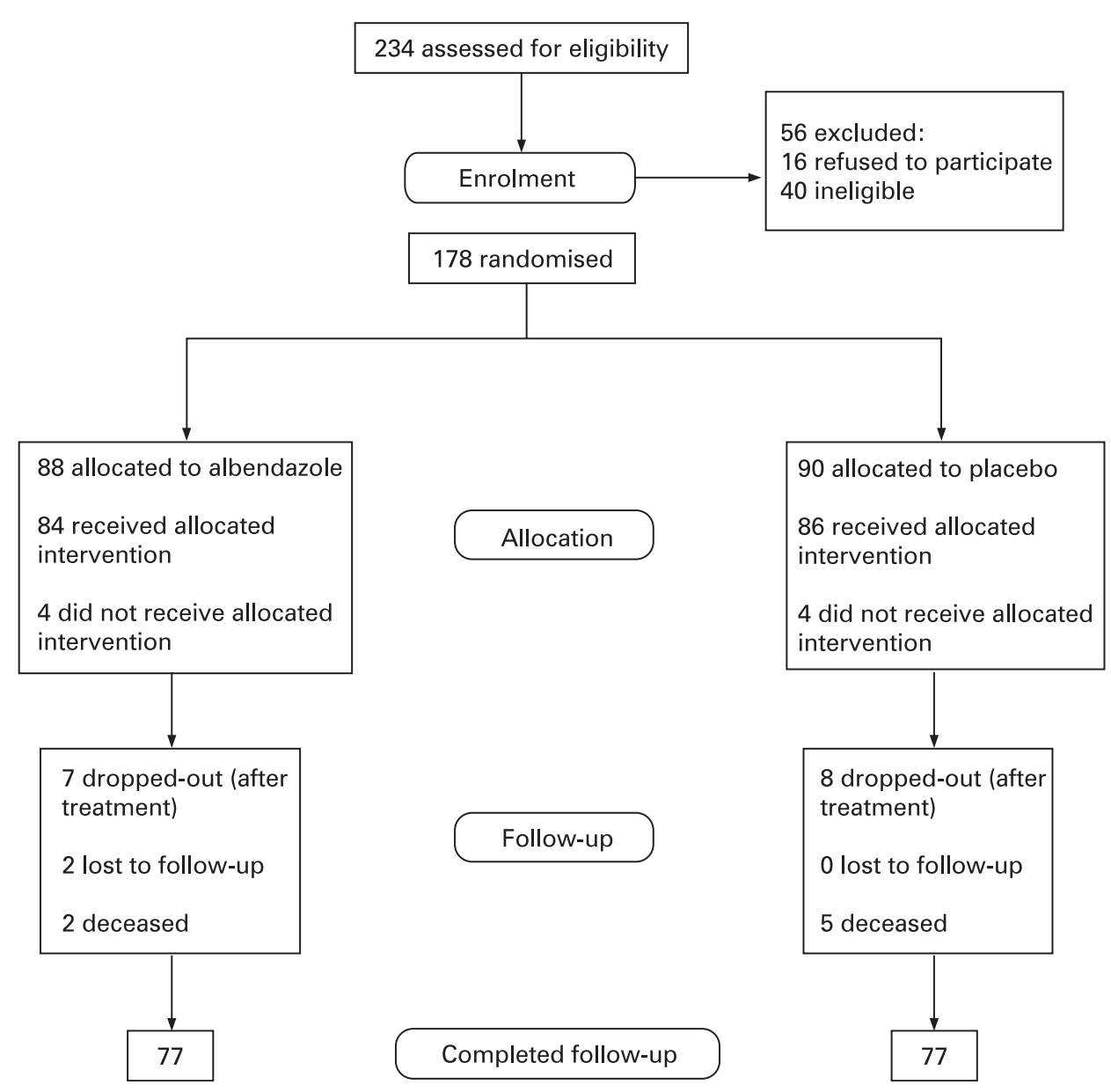


Table 1 Characteristics of the patients at baseline

\begin{tabular}{lcc}
\hline Characteristic & $\begin{array}{c}\text { Albendazole } \\
(\mathbf{n}=\mathbf{8 8})\end{array}$ & $\begin{array}{c}\text { Placebo } \\
(\mathbf{n}=\mathbf{9 0})\end{array}$ \\
\hline Demographic information & & \\
Age (y) (mean (SE)) & $39.39(17.79)$ & $41.55(16.80)$ \\
Children (<18 y) (No (valid \%)) & $8(9.2)$ & $7(8.0)$ \\
Males (No (valid \%)) & $51(58.6)$ & $46(52.9)$ \\
Females (No (valid \%)) & $36(41.4)$ & $41(47.1)$ \\
Missing age and gender information (n) & 1 & 3 \\
Cyst phase and location & & \\
Active cysts (No (valid \%)) & $59(68.6)$ & $57(66.3)$ \\
Transitional (No (valid \%)) & $49(57.0)$ & $54(66.8)$ \\
Calcifications (No (valid \%)) & $38(44.2)$ & $32(37.2)$ \\
Parenchymal cysts (No (valid \%)) & $74(86.0)$ & $74(82.2)$ \\
$\quad$ Active parenchymal cysts & $45(52.3)$ & $39(45.3)$ \\
Transitional parenchymal cysts & $40(46.5)$ & $52(60.5)$ \\
$\quad$ Calcified parenchymal cysts & $35(40.7)$ & $31(36.0)$ \\
Extraparenchymal cysts (No (valid \%)) & $35(40.7)$ & $42(48.8)$ \\
$\quad$ Active extraparenchymal cysts & $28(32.6)$ & $30(34.9)$ \\
Transitional extraparenchymal cysts & $1112.8)$ & $1315.1)$ \\
Calcified parenchymal cysts & $7(8.1)$ & $7(8.1)$ \\
Missing baseline scan (n) & 2 & 4 \\
Most common symptoms & & \\
Headache (No (valid \%)) & $60(68.2)$ & $64(71.1)$ \\
Seizures (No (valid \%)) & $51(59.3)$ & $56(64.4)$ \\
Dizziness (No (valid \%)) & $16(18.6)$ & $28(32.2)$ \\
Gait problems (No (valid \%)) & $13(15.1)$ & $17(19.5)$ \\
Limb weakness (No (valid \%)) & $13(15.1)$ & $9(10.3)$ \\
Depression and/or anxiety (No (valid \%)) & $15(15.1)$ & $17(19.5)$ \\
\hline Categora a not & &
\end{tabular}

${ }^{*}$ Categories are not mutually exclusive; patients may have had cysts of more than one type and in more than one location and may have experienced multiple symptoms.

to good, ${ }^{16}$ with kappa values ranging from 0.4 to 0.7 for identifying that a patient had cysts in a specific location or phase.

\section{Sample size and randomisation}

Sample size was determined for the primary outcome (the proportion of patients free of active cysts at 6 months). A sample size of 270 patients was estimated to be necessary to have $90 \%$ power to detect a response rate difference of $18 \%$ or more between the two groups.

Patients were allocated to treatment group according to a stratified block randomisation scheme. Two strata were considered: centre (six centres) and location of the cyst (parenchymal vs extraparenchymal). Permuted blocks of size 4 and 6 were used to balance the treatment allocation within each stratum. The randomisation lists were kept in electronic form on a computer accessible only to the statistician. All other research staff were blinded to the treatment arm.

\section{Statistical methods}

All analyses were performed according to an intent to treat principle. Thus all patients randomised were included in the group to which they were initially assigned, regardless of adherence. All tests were conducted at the 0.05 two tailed significance level.

The primary outcome measure was disappearance of active cysts by 12 months of follow-up, evaluated through serial imaging studies. The $\chi^{2}$ test was used to compare the proportion of patients free of active cysts at 12 months. We also explored active cyst disappearance before 12 months and the possible interaction of drug treatment with the patient's weight (as a continuous variable and as a categorical variable defined as $\leqslant 60 \mathrm{~kg}$ ) in predicting active cyst disappearance using both stratified analysis and logistic regression.

Secondary outcomes included disappearance of transitional or calcified cysts at 1, 6 and 12 months, change in number of cysts in a specific phase, time to seizure recurrence and adverse events. For examining secondary outcomes, we used the Wilcoxon rank sum test to look at the change in the number of cysts between follow-up images. Kaplan-Meier curves and the log rank test were used to estimate time to seizure recurrence in the two treatment groups ${ }^{17}$; the $\chi^{2}$ or Fisher's exact test was used to evaluate differences in potential adverse events during treatment and for the first month following treatment.

\section{Follow-up}

After treatment completion, patients were seen at 2 weeks, 1 month and every 3 months thereafter. Repeat scans were performed at 1 month, 6 months and 1 year after study entry.

\section{RESULTS \\ Participants}

Between February 2001 and February 2003, 178 patients with NC agreed to participate in the study; 88 patients were randomised to the ALB group and 90 to the placebo group (fig 1). Follow-up continued until February 2005.

Baseline data for all randomised patients is provided in table 1 by treatment group. Age range was 3-82 years (mean 39.4 years). Only 15 (9\%) of the patients were less than 18 years old. Fifty-six per cent of the patients were men. After taking into account early withdrawals (eight patients withdrew after randomisation but before taking study medication), there were 84 cases in the active treatment arm and 86 cases in the placebo treatment arm (fig 1). Of the 107 patients with new onset seizure as a symptom at baseline, 51 (48\%) were randomised to active treatment and 56 (52\%) were randomised to receive placebo. Patients randomised to ALB had a greater number of calcifications at baseline $(p=0.018)$, but the frequency of lesions (active and transitional) and their location (parenchymal and extraparenchymal) were similar in the two treatment groups. Seventy-seven patients in each arm completed the entire 2 year follow-up period (fig 1).

\section{Outcomes}

\section{Freedom from cysts}

Active cysts were identified in 59 (69\%) of the 86 people randomised to ALB and 57 (66\%) of the 86 people in the placebo arm (table 2). By 12 months following treatment, 38\% (20/53) of those with 12 month scans were free of active cysts in the treatment group compared with $20 \%(10 / 50)$ in the placebo group $(p=0.048)$. This difference in cyst disappearance by treatment was greatest at 1 month of follow-up, with 31\% (18/ 58 ) of those in the ALB group being free of active cysts at month 1 of follow-up compared with $7 \%(4 / 55)$ of those in the placebo group ( $p=0.001)$. Of those patients followed and scanned at 6 months, $35 \%(18 / 51)$ were free of active cysts in the treatment arm compared with $12 \%(6 / 50)$ in the placebo group $(p=0.006)$. A similar trend was seen when looking at active parenchymal and extraparenchymal cysts separately, but the association was not statistically significant for extraparenchymal cysts (table 2). There was no significant interaction between the drug and the participant's weight in predicting the disappearance of active cysts. 
Table 2 Number (valid \%) of patients in whom cysts disappeared out of the total who had cysts of that type at baseline (\%)

\begin{tabular}{|c|c|c|c|c|c|c|c|c|c|}
\hline & \multicolumn{3}{|l|}{ Active cysts } & \multicolumn{3}{|c|}{ Transitional cysts } & \multicolumn{3}{|l|}{ Calcifications } \\
\hline & Albendazole & Placebo & $\begin{array}{l}\text { p Value } \\
\text { for } \chi^{2} \\
\text { test }\end{array}$ & Albendazole & Placebo & $\begin{array}{l}p \text { Value } \\
\text { for } \chi^{2} \\
\text { test }\end{array}$ & Albendazole & Placebo & $\begin{array}{l}\text { p Value } \\
\text { for } \chi^{2} \\
\text { test }\end{array}$ \\
\hline Baseline & $0 / 59(0)$ & $0 / 57(0)$ & Referent & $0 / 49(0)$ & $0 / 54(0)$ & Referent & $0 / 38(0)$ & $0 / 32(0)$ & Referent \\
\hline 1 month & $18 / 58(31.0)$ & $4 / 55$ (7.3) & 0.001 & $13 / 47(27.0)$ & $12 / 52(23.1)$ & 0.600 & $30 / 37(81.1)$ & $20 / 30(66.7)$ & 0.178 \\
\hline 6 month & $18 / 51$ & $6 / 50(12.0)$ & 0.006 & $22 / 42(52.4)$ & $19 / 49(38.8)$ & 0.194 & $28 / 34(82.4)$ & $18 / 28(64.3)$ & 0.106 \\
\hline Baseline & $45 / 45(0)$ & $39 / 39(0)$ & Referent & $40 / 40(0)$ & $52 / 52(0)$ & Referent & & & \\
\hline 1 month & $18 / 44(40.9)$ & 4/37 (10.8) & 0.002 & $11 / 38(29.0)$ & $14 / 50(28.0)$ & 0.922 & & & \\
\hline 6 month & $19 / 39(48.7)$ & $8 / 27(22.9)$ & 0.021 & $19 / 34(55.9)$ & $21 / 47(44.7)$ & 0.320 & & & \\
\hline 12 month & $20 / 41$ (48.8) & 7/36 (19.4) & 0.007 & $20 / 34(58.8)$ & $29 / 49(59.2)$ & 0.974 & & & \\
\hline \multicolumn{10}{|c|}{ Extraparenchymal } \\
\hline Baseline & $28 / 28(0)$ & $30 / 30(0)$ & Referent & $11 / 11(0)$ & $13 / 13(0)$ & Referent & & & \\
\hline
\end{tabular}

*Fisher's exact test.

Transitional cysts were identified in $49(57 \%)$ of the 86 people randomised to ALB and in $54(63 \%)$ of the 86 people in the placebo arm (table 2). By 1 month following treatment, $27 \%$ (13/47) of those with 1 month scans were free of transitional cysts in the treatment group compared with $23 \%(12 / 52)$ in the placebo group $(p=0.600)$. Of those patients followed and scanned at 6 months, $52 \%(22 / 42)$ were free of transitional cysts in the treatment arm compared with 39\% (19/49) in the placebo group $(p=0.194)$. After 1 year of follow-up, 55\% (23/ 42) of those in the treatment arm were free of transitional cysts compared with $53 \%(27 / 51)$ in the placebo group $(p=0.861)$. There was also no significant difference in the disappearance of transitional cysts by drug treatment in the subanalyses by brain location (parenchymal or extraparenchymal) (table 2).

\section{Reduction in the number of cysts}

The number of active cysts apparent on a patient's CT or MRI during the study ranged from 0 to 36 , excluding patients with too many active cysts to count (eight excluded at baseline, seven at months 1 and 6 , and six excluded at month 12). The mean number of active cysts decreased between baseline and month 1 for the ALB (mean number at baseline 3.88 and at 1 month 1.86) group but not for the placebo group (mean at baseline 2.67 and at 1 month 2.69). We looked at the change in the number of cysts of each phase overall and by location in the brain (parenchymal or extraparenchymal) using the Wilcoxon

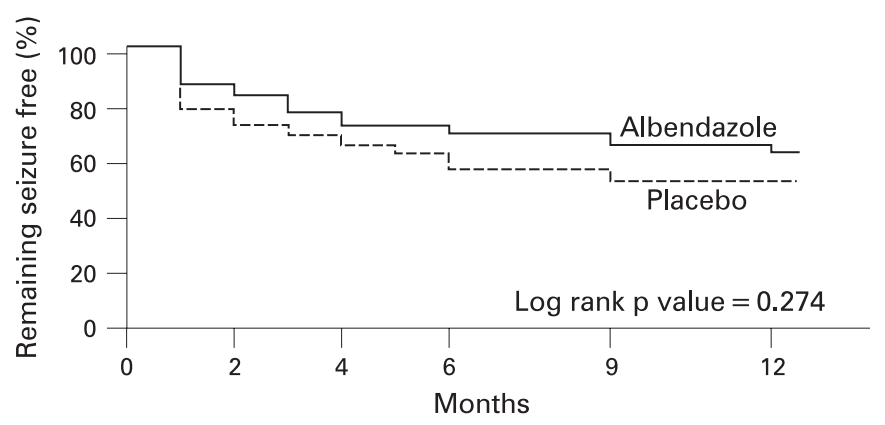

Figure 2 Per cent of patients with seizure at baseline who survived seizure free after treatment with albendazole or placebo over time rank sum test. Those taking ALB had a significant decrease in the number of active cysts between baseline and month 1 compared with those in the placebo group $(p=0.001)$. This trend is consistent when looking at all active cysts and only active parenchymal cysts $(p=0.002)$ or active extraparenchymal cysts $(p=0.021)$. There was no difference by treatment group in the change in the number of active cysts between month 1 and month $6(p=0.797)$ or month 6 and month 12 of follow-up $(p=0.938)$.

The number of transitional cysts apparent on a patient's CT or MRI during the study ranged from 0 to 38 , after excluding those with too many transitional cysts to count (four excluded at baseline, five at month 1 and four at months 6 and 12). The total number of calcifications apparent on a patient's CT or MRI ranged from 0 to 45 , excluding those with too many calcified cysts to count (six excluded at baseline, two at month 1 , three at month 6 and four at month 12). The change in the number of transitional cysts and inactive calcifications between baseline and month 1 of follow-up did not differ by treatment group ( $p$ value for transitional cysts $0.234, p$ value for calcifications 0.456 ).

\section{Seizure freedom}

At baseline, 107 patients reported new onset of seizure. Of these, 51 (48\%) were randomised to the ALB group and 56 $(52 \%)$ to the placebo group. Using Kaplan-Meier survival analysis, the proportion of patients with seizure at baseline who were seizure free at 12 months of follow-up was 0.62 in the ALB group and 0.52 in the placebo group (fig 2). The mean time seizure free was 8.86 months in the ALB group versus 7.67 months in the placebo group; this difference was not statistically significant $(p=0.274)$. Findings were similar to those with only parenchymal cysts $(p=0.318)$ and those with any extraparenchymal cysts (with or without parenchymal cysts) ( $p=0.998)$. It was not possible to evaluate seizure outcome for those with only extraparenchymal cysts because the number with seizures at baseline was small (four patients).

\section{Possible adverse events}

The three most common symptoms reported during treatment and the first month following treatment were headache, 
Table 3 Number of patients with possible adverse events by treatment (\% of valid responses to question)

\begin{tabular}{lcll}
\hline & $\begin{array}{l}\text { No (valid \%) with } \\
\text { symptom in } \\
\text { albendazole group }\end{array}$ & $\begin{array}{l}\text { No (valid \%) } \\
\text { with symptom in } \\
\text { placebo group }\end{array}$ & $\begin{array}{l}\text { p Value for } \\
\chi^{2} \text { test }\end{array}$ \\
\hline Symptom & $2(2.4)$ & $3(3.5)$ & $1.00^{*}$ \\
\hline $\begin{array}{l}\text { During } 8 \text { days of treatment } \\
\text { Seizures }\end{array}$ & $59(70.2)$ & $53(61.6)$ & 0.236 \\
$\quad \begin{array}{l}\text { Headache } \\
\text { Stomach problems } \\
\text { (nausea, pain or vomiting) }\end{array}$ & $38(45.2)$ & $40(46.5)$ & 0.868 \\
$\begin{array}{l}\text { Intracranial hypertension } \\
\text { During first month following treatment }\end{array}$ & $0(0)$ & $3(0.5)$ & $0.246^{*}$ \\
$\quad$ Seizures & & & \\
$\quad \begin{array}{l}\text { Headache } \\
\text { Stomach problems } \\
\text { (nausea, pain or vomiting) }\end{array}$ & $8(9.8)$ & $10(12.0)$ & 0.637 \\
Intracranial hypertension & $50(61.0)$ & $51(61.4)$ & 0.951 \\
\hline *Fisher's exact test. & $9(11.0)$ & $13(15.7)$ & 0.376 \\
& $0(0)$ & $0(0)$ & $\mathrm{NA}$ \\
\hline
\end{tabular}

*Fisher's exact test.

seizures and stomach problems (table 3). During the 8 days of treatment, three patients developed intracranial hypertension, all in the placebo group (table 3 ).

During the 2 year study period, seven people died, all but one due to cysticercosis. Two of the deaths occurred in the treatment group and five in the placebo group (fig 1). This difference was not statistically significant (Fisher's exact test $p=0.213$ ). All but one of the deceased patients presented with the extraparenchymal form of NC, and four of the six had been shunted more than 1 year prior to study enrolment. Repeat shunting was performed in four patients with generally poor outcome. Shunting of NC patients is generally associated with a high shunt failure rate ${ }^{18}$ and high mortality. ${ }^{19} 20$

\section{DISCUSSION}

Our primary analysis of active cyst disappearance by 12 months revealed a clear advantage for the ALB group compared with placebo, and the effect occurred within the first 30 days following initiation of treatment. There seems to be no additional advantage in the treatment group compared with placebo after this time. The reduction in the number of active cysts was also most pronounced at 1 month. Nonetheless, about $70 \%$ of people are not rendered free of active cysts with this first course of therapy. These results are similar to those reported by Garcia and colleagues. ${ }^{5}$ At 6 months after treatment, active cysts disappeared in 38\% of patients receiving ALB plus steroids compared with $15 \%$ of patients receiving placebo alone. We also confirm previous reports regarding the lack of effectiveness of ALB treatment on transitional or degenerative cysts. $^{21-25}$

About half of the people with seizures at baseline remained seizure free at 1 year following initiation of treatment, and there was no significant effect of treatment with ALB on this outcome. This finding is similar to that obtained in our previous trial $^{26}$ two observational studies ${ }^{27}$ and two trials involving paediatric patients. ${ }^{28}{ }^{29}$ However, several studies have found a significant reduction in seizures in patients treated with ALB, including two clinical trials with children, ${ }^{21}{ }^{30}$ and a meta analysis. ${ }^{11}$ A recent trial in adult patients did not find a reduction in the number of seizures overall, but did report a significant reduction in the number of generalised seizures in patients treated with ALB and steroids compared with those treated with only placebo. ${ }^{5}$ Thus the findings regarding the influence of ALB on seizure recurrence are inconsistent.
However, there is no indication that ALB treatment increases the risk of seizures or any other adverse event. Symptoms during treatment and for the first month following treatment were similar in the ALB and placebo groups.

An important question that remains is the effect of ALB on extraparenchymal cysts. The association of treatment with the disappearance of active extraparenchymal cysts was not significant, although there was the suggestion of a trend. We did find a significant reduction in the number of active extraparenchymal cysts in the ALB group compared with the placebo group between baseline and month 1 . This difference in the results by outcome (disappearance versus number of active cysts) may be due to a lack of statistical power to address cyst disappearance.

If the objective of treatment is to eradicate active cysts, it would appear that a single course of therapy with ALB (the usual practice) provides benefit over placebo in only $24 \%$ of patients. Since $69 \%$ of patients receiving ALB continue to demonstrate active cysts, new drugs or different treatment regimens need to be developed for this majority of patients with NC. Our finding that not all encysted parasites die after a single course of antiparasitic treatment was also described in a recent literature review. ${ }^{31}$ Treatment options to be explored may include a higher initial dose of drug, concomitant or subsequent treatment with an AHD that has a different mode of action, such as praziquantel, or a repeat course of ALB or of another AHD in those with persistent active cysts at 1 month. We also need studies to explore reasons for the heterogeneous effect of ALB to guide the development of treatment strategies for the large proportion of patients who do not seem to benefit from ALB.

Acknowledgements: We are indebted to Drs Eduardo Castro, Gastón Rodríguez, Iván Reinoso, Victor Paredes and Carlos Barrionuevo of the "Carlos Andrade Marín" Hospital of Quito, Ecuador, for support in patient care, and to Dr Franklin Santillán of the Social Security Hospital of Cuenca, Ecuador, for patient referrals. Other members of the Neurocysticercosis Clinical Trial Group who collaborated in this study include: Karina Quinde, Research Assistant; Ana Montenegro, Research Social Worker; Alicia Vasco, Research Nurse; Mery Obregon, Research Nurse; Luis Villalta, Accountant; Nora Hernandez, MD, Project Coordinator; Mary Angie Garcia, Research Assistant. The authors of this manuscript participated in the study design and implementation, as well as in the data analysis and/or manuscript preparation.

Funding: This study was supported by NINDS grant \#R01-NS39403; Glaxo/SKB and Acromax Co supplied active drug and placebo.

Competing interests: None. 
Ethics approval: Approval for the study was granted by the Institutional Review Board of Columbia University, the Office for Human Research Protection (OHRP) of the National Institutes of Health in the USA, as well as the ethics committees at each of the participating hospitals.

The Ecuadorian Neurocysticercosis Group: Noemi Lisanti, I"Comprehensive Epilepsy Center", Cuenca), Rafael Aguirre, ("Teodoro Maldonado" Hospital, Guayaquil), Marcos Serrano, ("Carlos Andrade Marín" Hospital, Quito), Jorge Pesantes, Jorge Moncayo,

("Eugenio Espejo" Hospital, Quito) and Marcelo Roman, ("Baca Ortiz" Hospital, Quito).

\section{REFERENCES}

1. White A. Neurocysticercosis. Curr Treat Options Infect Dis 2000:2:78-87.

2. Carpio A, Hauser W. Prognosis for seizure recurrence in patients with newly diagnosed neurocysticercosis. Neurology 2002;59:1730-4.

3. Hawk M, Shahlaie K, Kim K, et al. Neurocysticercosis in the United States: review of an important emerging infection. Neurology 2004;63:1559-64.

4. Schantz P. Taenia solium cysticercosis: an overview of global distribution and transmission. Oxon, UK: CABI Publishing, 2002:63-74.

5. Garcia $\mathbf{H}$, Pretell E, Gilman R, et al. A trial of antiparasitic treatment to reduce the rate of seizures due to cerebral cysticercosis. N Engl J Med 2004:350:249-58.

6. Singh G, Sander J. Anticysticercal treatment and seizures in neurocysticercosis Lancet Neurol 2004;3:207-8.

7. Takayanagui 0. Therapy for neurocisticercosis. Expert Rev Neurother 2004;4:129-39.

8. Caplan L, Estanol B, Mitchel W, et al. How to manage patients with neurocysticercosis. Eur Neurol 2997;37:124-31.

9. Salinas R, Prasad K. Drugs for treating neurocysticercosis (tapeworm infection of the brain). Oxford: Update Software, 2005.

10. Carpio A, Escobar A, Hauser W. Cysticercosis and epilepsy: a critical review. Epilepsia 1998;39:1025-40.

11. Del Brutto 0, Roos K, Coffey C, et al. Meta-analysis: Cysticidal drugs for neurocysticercosis: albendazole and praziquantel. Ann Intern Med 2006;145:43-51.

12. Evans C, Garcia H, Gilman R, et al. Controversies in the management of cysticercosis. Emerg Infect Dis 1997;3:403-5.

13. Carpio A, Placencia M, Santillán F, et al. A proposal for a classification of neurocysticercosis. Can J Neuro Sci 1994;21:43-7.

14. Garcia HH, Gilman RH, Horton J, et al. Albendazole therapy for neurocysticercosis: a prospective double-blind trial comparing 7 versus 14 days of treatment. Cysticercosis Working Group in Peru. Neurology 1997:48:1421-7.
15. GlaxoSmithKline. ALBENZA. Available at: http://us.gsk.com/products/assets/ us albenza.pdf (accessed 24 June 2008).

16. Fleiss J. Statistical methods for rates and proportions. New York: John Wiley \& Sons, 1981:218.

17. Kaplan E, Meier P. Nonparametric estimation from incomplete observations. JASA 1958; 53:457-81.

18. Kelley R, Duong D, Locke G. Characteristics of ventricular shunt malfunctions among patients with neurocysticercosis. Neurosurgery 2002;50:757-62.

19. Colli B, Pereira C, Assirati JJ, et al. Isolated fourth ventricle in neurocysticercosis: pathophysiology, diagnosis, and treatment. Surg Neurol 1993;39:305-10.

20. McCormick G. Cysticercosis-review of 230 patients. Bull Clin Neurosci 1985:50:76-101.

21. Singhi P, Jain V, Khandelwal N. Corticosteroids versus albendazole for treatment of single small enhancing computed tomographic lesions in children with neurocysticercosis. J Child Neurol 2004:19:323-7.

22. Padma M, Behari M, Misra N, et al. Albendazole in single CT ring lesions in epilepsy. Neurology 1994;44:1344-6.

23. Rajshekhar V. Rate of spontaneous resolution of a solitary cysticercus granuloma in patients with seizures. Neurology 2001;57:2315-17.

24. Gogia S, Talukdar B, Choudhury V, et al. Neurocysticercosis in children: clinical findings and response to albendazole therapy in a randomized, double-blind, placebo-controlled trial in newly diagnosed cases. Trans $R$ Soc Trop Med Hyg 2003;97:416-21.

25. Garcia H, Evans C, Nash T, et al. Current consensus guidelines for treatment of neurocysticercosis. Clin Microbiol Rev 2002;15:747-56.

26. Carpio A, Santillán F, León P, et al. Is the course of neurocysticercosis modified by treatment with antihelminthic agents? Arch Int Med 1995:155:1982-8.

27. Rajshekhar V, Lakshmanan J. Seizure outcome in patients with a solitary cerebral cysticercus granulomas. Neurology 2004;62:2236-40.

28. Baranwal AK, Singhi PD, Khandelwal N, et al. Albendazole therapy in children with focal seizures and single small enhancing computerized tomographic lesions: a randomized, placebo-controlled, double blind trial. Pediatr Infect Dis J 1998:17:696-700.

29. Gogia S, Talukdar B, Choudhury V, et al. Neurocysticercosis in children: clinical findings and response to albendazole therapy in a randomized, double-blind, placebo-controlled trial in newly diagnosed cases. Trans $R$ Soc Trop Med Hvg 2003:97:416-21.

30. Kalra V, Dua T, Kumar V. Efficacy of albendazole and short-course dexamethasone treatment in children with 1 or 2 ring-enhancing lesions of neurocysticercosis: a randomized controlled trial. J Pediatr 2003:143:111-14.

31. Nash T, Singh G, White A, et al. Treatment of neurocysticercosis: current status and future research needs. Neurology 2006;67:1120-7. 